\title{
MicroRNA-302d downregulates TGFBR2 expression and promotes hepatocellular carcinoma growth and invasion
}

\author{
YUE-LIANG CHEN $^{1}$, QIU-PING XU ${ }^{1}$, FENG GUO $^{1}$ and WEN-HUA GUAN ${ }^{2}$ \\ ${ }^{1}$ Department of Critical Care Medicine, Sir Run Run Shaw Hospital of Zhejiang University, Hangzhou, Zhejiang 310016; \\ ${ }^{2}$ Department of Infectious Diseases, The First Affiliated Hospital of Zhejiang University, \\ Hangzhou, Zhejiang 310003, P.R. China
}

Received August 26, 2015; Accepted September 22, 2016

DOI: $10.3892 /$ etm.2016.3970

\begin{abstract}
Hepatocellular carcinoma (HCC) is the second leading cause of cancer-associated mortality in China and the third leading cause worldwide. A number of microRNAs (miRNAs) have been implicated in cell cycle progression, growth, apoptosis, angiogenesis and metastasis in HCC. In the present study, reverse transcription-quantitative polymerase chain reaction analysis was used to detect the levels of miR-302d expression in the tissues of 30 patients with HCC. Cell cycle, growth, apoptosis and migration were analyzed using a cell counting kit, flow cytometry and a Transwell migration assay. Dual-luciferase reporter assays and western blotting were also used to analyze the expression levels of transforming growth factor beta type II receptor (TGFBR2) in HCC cells. The present study evaluated the role of miR-302d in the development and progression of HCC. Abnormally high expression of miR-302d was observed in $80 \%$ of $\mathrm{HCC}$ specimens. Moreover, patients with lower levels of miR-302d expression experienced a longer survival time than those with higher levels of miR-302d expression. It was demonstrated that miR-302d promoted HCC cell growth and migration, suppressed cell apoptosis and affected cell cycle distribution in vitro, and augmented tumorigenicity in vivo. Furthermore, TGFBR2, which is a tumor suppressor, was confirmed as a target of miR-302d in HCC cells. Dual-luciferase reporter assays indicated that TGFBR 2 expression was negatively regulated by miR-302d. Taken together, the results of the present study suggest that miR-302d may serve as a valuable tool for predicting the prognosis of patients with HCC.
\end{abstract}

Correspondence to: Dr Wen-Hua Guan, Department of Infectious Diseases, The First Affiliated Hospital of Zhejiang University, 79 Qingchun Road, Hangzhou, Zhejiang 310003, P.R. China E-mail: guanwhhz@163.com

Key words: microRNA-302d, hepatocellular carcinoma, tumorigenicity, tumor promoter, transforming growth factor beta type II receptor

\section{Introduction}

Hepatocellular carcinoma (HCC) has been ranked as the second leading cause of cancer-associated mortality in China and the third leading cause of cancer mortality worldwide $(1,2)$. Incidence and mortality rates of HCC continue to increase in various countries across the world, including the United States (3). Primary causes of HCC include alcohol consumption, dietary exposure to aflatoxin B1 and infection with hepatitis virus (4). Although numerous risk factors have been identified, the pathogenetic mechanisms underlying HCC development and progression remain unclear. Investigating the molecular mechanisms that contribute to HCC may enable the development of novel therapeutic strategies and improve patient prognosis.

MicroRNAs (miRNAs) are endogenous small noncoding RNAs and act as oncogenes or tumor suppressors in cancer (5). Previous studies investigating HCC have demonstrated that miRNA expression profiles are signatures of HCC development and progression (6-8). Various miRNAs, including miR-302d, have been implicated in the onset and development of HCC (9). The identification of novel functions of specific miRNAs may help elucidate the molecular mechanisms that underlie HCC development and progression.

It has been reported that miR-302d is highly expressed in human embryonic stem cells and, as such, it is recognized as a human embryonic stem cell-specific miRNA (10). miR-302d directly contributes to the regulation of p21 expression and governs the $G_{1} / S$ transition checkpoint in human embryonic stem cells (11). By inhibiting p21, miR-302d rescues Ras-induced senescence in human mammary epithelial cells (12). Additionally, miR-302d increases cell proliferation and inhibits cell death induced by oxidizing agents in human adipose tissue-derived mesenchymal stem cells (13). Increasing evidence suggests that embryogenesis and tumorigenesis involve the activation of the same pathways; therefore, miR-302d may perform the same functions during tumorigenesis as occurs during embryogenesis (14). However, the role of miR-302d in HCC remains unclear. The microarray expression profile of $\mathrm{HCC}$ indicates that miR-302d is highly expressed in HCC in the present study (data not shown). In the present study, in vitro and in vivo studies were performed to determine whether miR-302d promotes the proliferation and migration 
of HCC and enhances tumor growth, by investigating the association between miR-302d and transforming growth factor beta type II receptor (TGFBR2).

\section{Materials and methods}

Patients and tissue samples. A total of 140 patients with stage I-IV HCC admitted to the Department of Infectious Diseases, First Affiliated Hospital of Zhejiang University (Hangzhou, China) between February 2011 and October 2014 were enrolled in the present study. The inclusion criteria of the current study were that no patients had received radiotherapy or chemotherapy. Complete clinical and pathological follow-up data was available for 110 patients, who were grouped into two groups according to their median levels of miR-302d expression [high expression of miR-302d $(n=55)$ vs. low expression of miR-302d ( $\mathrm{n}=55)]$ and the association between miR-302d expression and the survival of patients following surgery was examined. The survival of HCC patients was defined as 0 and mortality of HCC patients was defined as 1, and the survival time of patients was analyzed using GraphPad Prism software ver. 5.0 (GraphPad Software, Inc., La Jolla, CA, USA). Specimens of HCC and paired non-cancerous tissues from surrounding adjacent areas were obtained from 30 patients via surgical resection. All tissues were snap frozen in liquid nitrogen immediately after resection to be used in subsequent experiments to measuremiR-302d expression. Ethical approval for the present study was provided by the Independent Ethics Committee of The First Affiliated Hospital of Zhejiang University. Informed and written consent was obtained from all patients or their family members in accordance with the Ethics Committee guidelines.

Cell culture and transfection. Cells from the SMMC-7721 human hepatocellular carcinoma cell line were purchased from JRDUN Biotechnology Co., Ltd., (Shanghai, China) and maintained in RPMI 1640 medium supplemented with $10 \%$ fetal bovine serum (FBS; Gibco; Thermo Fisher Scientific, Inc., Waltham, MA, USA) at $37^{\circ} \mathrm{C}$ in a humidified chamber containing $5 \% \mathrm{CO}_{2}$. miR-302d mimics and inhibitors were purchased from Shanghai GenePharma Co., Ltd., (Shanghai, China). For transient transfection of cells in 6-well plates, $100 \mu \mathrm{M}$ mimics or inhibitors were added with Lipofectamine 2000 reagent (Invitrogen; Thermo Fisher Scientific, Inc.) in OPTI-MEM media (Gibco; Thermo Fisher Scientific, Inc.) according to the manufacturer's instructions.

$R N A$ isolation and reverse transcription-quantitative polymerase chain reaction (RT-qPCR) analysis. Total RNA was isolated from normal and HCC tissue using TRIzol reagent (Invitrogen; Thermo Fisher Scientific, Inc.) according to the manufacturer's protocol. cDNA was synthesized from RNA using a MMLV RT reagent kit (Thermo Fisher Scientific, Inc.), according to the manufacturer's protocol. A specific primer (5'-CTCAACTGGTGTCGTGGAGTCGGCAATTCAGTT GAGCCACACTC-3') was used to synthesize miR-302d cDNA. cDNA were amplified using a SYBRGreen PCR kit (Thermo Fisher Scientific, Inc.). PCR primers for miR-302d were as follows: Forward, 5'-ACAGTGTCGTTCACGGAGGT-3' and reverse, 5'-AACTGGTGTCGTGGAGTCGGC-3'. PCR amplification was performed on an Applied Biosystems 7500 real-time PCR platform (Thermo Fisher Scientific, Inc.). The PCR cycling conditions were as follows: $95^{\circ} \mathrm{C}$ for $10 \mathrm{~min}$, followed by 40 cycles at $95^{\circ} \mathrm{C}$ for $15 \mathrm{sec}$ and $60^{\circ} \mathrm{C}$ for $45 \mathrm{sec}$, and a final extension step of $95^{\circ} \mathrm{C}$ for $15 \mathrm{sec}, 60^{\circ} \mathrm{C}$ for $1 \mathrm{~min}$, $95^{\circ} \mathrm{C}$ for $15 \mathrm{sec}$ and $60^{\circ} \mathrm{C}$ for $15 \mathrm{sec}$. The experiment was repeated three times. To normalize miR-302d, 5S rRNA was used as an internal standard. PCR primers for 5S rRNA were as follows: Forward, 5'-CCATACCACCCTGGAAACGC-3' and reverse, 5'-TACTAACCGAGCCCGACCCT-3'. Relative quantification of miR-302d expression levels was determined using the $2^{-\Delta \Delta \mathrm{Cq}}$ method (15).

Cell growth analysis. Cells were seeded in a 96-well plate $\left(1 \times 10^{5}\right.$ cells/well) and cultured at $37^{\circ} \mathrm{C}$ for $24 \mathrm{~h}$. Cells were subsequently transfected with miRNA mimics, antisense or negative controls (NC; untreated cells) for $72 \mathrm{~h}$. Cell growth was assessed every $24 \mathrm{~h}$ using a cell counting kit (CCK)-8 assay (Beyotime Institute of Biotechnology, Shanghai, China). All samples were evaluated in triplicate for each group and the experiment was repeated at least twice.

Cell cycle analysis. To determine cell cycle properties, cells were transfected with miRNA mimics, antisense or NC. After $36 \mathrm{~h}$, transfected cells were harvested by trypsinization and incubated with $0.5 \mu \mathrm{g} / \mathrm{ml}$ RNase A (Thermo Fisher Scientific, Inc.) and $100 \mu \mathrm{g} / \mathrm{ml}$ propidium iodide (PI) for $30 \mathrm{~min}$ at room temperature prior to fluorescence-activated cell sorting with a flow cytometer (BD Biosciences, Franklin Lakes, NJ, USA).

Apoptosis assay. For the analysis of apoptosis, cells were seeded into 6-well plates and transfected with miRNA mimics, antisense or NC. At $48 \mathrm{~h}$ after transfection, cells were collected, washed and stained using the Annexin V fluorescein isothiocyanate/PI double staining kit (BD Biosciences) according to the manufacturer's instructions. A flow cytometer was used to analyze apoptotic cells (BD Biosciences).

In vitro migration assays. Cell migration was assessed using Transwell insert chambers (Corning, Inc., Corning, NY, USA). Cells transfected with miRNA mimics, antisense or NC were re-suspended in $100 \mu \mathrm{l}$ RPMI1640 and placed into the upper chamber without Matrigel. RPMI1640 supplemented with $10 \%$ FBS was added to the lower chamber and functioned as the chemoattractant. Cells were incubated for $24 \mathrm{~h}$. Migrated cells were fixed with $4 \%$ polyoxymethylene, stained with $0.2 \%$ crystal violet and imaged under an inverted microscope (Olympus Corp., Tokyo, Japan) at a magnification of x200.

Tumor growth assay. A total of 12 male BALB/c nude mice (15-18 g), aged 4-5 weeks old, were purchased from Shanghai SLAC Laboratory Animal Co., Ltd., (Shanghai, China). Mice were equally divided into two groups $(n=6)$ and received a subcutaneous injection in their right flank of either $1 \times 10^{7}$ SMMC-7721 cells stably expressing miR-302d inhibitor or SMMC-7721 cells without treatment (the NC). Mice were housed in the animal facility at a temperature of $25^{\circ} \mathrm{C}$ and humidity of $60-70 \%$ and subjected to a 12 -h light-dark cycle with ad libitum access to food and water. Mice were observed over 6 weeks for tumor formation. Mice 
were subsequently sacrificed by intraperitoneal injection of $3 \%$ sodium pentobarbital (40 mg/kg; Sigma-Aldrich; Merck Millipore, Darmstadt, Germany) and cervical dislocation was performed. The tumors were subsequently resected and the wet weights of each tumor were measured.

Western blot analysis. SMMC-7721 cells transfected with miRNA mimics, antisense or NC were lysed using radioimmunoprecipitation assay buffer supplemented with protease inhibitor (Beyotime Institute of Biotechnology). Protein concentration was estimated using the BCA Protein assay kit (Pierce; Thermo Fisher Scientific, Inc.). Equal amounts of protein $(20 \mu \mathrm{g})$ were separated by $12 \%$ SDS-PAGE and transferred to nitrocellulose membranes (EMD Millipore, Billerica, MA, USA). Following blocking with fat-free milk for $1 \mathrm{~h}$ at $25^{\circ} \mathrm{C}$, membranes were immunoblotted overnight at $4^{\circ} \mathrm{C}$ with primary antibodies against GAPDH (cat. no. 2251-1; 1:1,500; Fermentas; Thermo Fisher Scientific, Inc.) and TGFBR2 (cat. no. ab61213; 1:800; Abcam, Cambridge, MA, USA). Following washing, membranes were incubated with horseradish peroxidase-conjugated goat anti-rabbit immunoglobulin (Ig) G (cat. no. A0208; 1,000; Beyotime Institute of Biotechnology) and goat anti-mouse IgG (cat. no. A0216; 1:1,000; Beyotime Institute of Biotechnology) secondary antibodies at $37^{\circ} \mathrm{C}$ for $1 \mathrm{~h}$. Signals were detected using an enhanced chemiluminescence Western Blotting substrate (Pierce; Thermo Fisher Scientific, Inc.).

Luciferase reporter assays. TGFBR2 was predicted to interact with miR-302d by bioinformatics analysis using TargetScan (http://www.targetscan.org), which predicts biological targets of miRNAs by searching for the presence of 8 oligomer, 7 oligomer, and 6 oligomer sites that match the seed region of each miRNA (16). The 3'-untranslated region (UTR) of the human TGFBR2 gene predicted to interact with miR-302d was synthesized and inserted in the pGL3 vector (Promega Corp., Madison, WI, USA), downstream of the firefly luciferase gene to form pGL3-TGFBR2. SMMC-7721 cells ( $2 \times 10^{5} /$ well) were seeded in 96 -well plates, reporter plasmids were co-transfected with miR-302d mimics or NC using Lipofectamine 2000. Following 24 h, cells were lysed and the activities of Renilla and firefly luciferase were examined using the Dual-Luciferase Reporter assay system (Promega Corp.). Firefly luciferase activity was normalized to Renilla luciferase activity.

Statistical analysis. Statistical analysis was performed using GraphPad Prism software ver. 5.0 (GraphPad Software, Inc., La Jolla, CA, USA). Overall survival in relation to miR-302d expression was evaluated by Kaplan-Meyer survival curves and the log-rank nonparametric test. The results from different groups were compared using two-tailed Student's t-test. $\mathrm{P}<0.05$ was considered to indicate a statistically significant difference.

\section{Results}

Upregulated miR-302d expression is associated with poorer patient survival rates. The microarray expression profile of HCC indicated that miR-302d is highly expressed in HCC (data not shown). To further verify this finding, RT-qPCR was performed on 30 pairs of HCC tissue and adjacent healthy tissue samples. It was detected that levels of miR-302d expression were significantly higher in the HCC tissues compared with those in adjacent noncancerous tissue $(\mathrm{P}<0.001$; Fig. 1A). High expression of miR-302d ( $>1.50$-fold) was more frequently observed in HCC tissue $(24 / 30 ; 80 \%)$ than in noncancerous adjacent tissue (Fig. 1A). The HCC patients were divided into two groups according to the median level of miR-302d. Subsequently, the association between miR-302d expression and the survival of patients following surgery was examined. The results demonstrated that patients with lower levels of miR-302d expression experienced longer survival times than those with higher levels of miR-302d expression $(\mathrm{P}=0.015$; Fig. 1B).

miR-302d increases HCC cell growth in vitro. To determine the role of miR-302d in HCC progression, miR-302d mimics, inhibitors or NCs were transfected into SMMC-7721 cells (Fig. 2A). CCK-8 assays indicated that the viability of the miR-302d mimics group was significantly improved compared with the NC group $(\mathrm{P}<0.05)$, while the viability of the miR-302d inhibitor group was significantly reduced compared with the NC group $(\mathrm{P}<0.01$; Fig. 2B). Cell cycle distribution analysis demonstrated that miR-302d overexpressing SMMC-7721 cells exhibited a significantly decreased percentage of cells in the $G_{0} / G_{1}$ phase $(P<0.01)$ and a significant increase in the percentage of cells in the $\mathrm{S}$-phase, compared with NC-transfected cells $(\mathrm{P}<0.05$; Fig. $2 \mathrm{C})$. By contrast, in the miR-302d inhibitor group, there was a significant increase in the percentage of cells in the $G_{0} / G_{1}$ phase $(P<0.05)$ and a significant decrease in the percentage of cells in the $\mathrm{S}$-phase, compared with the NC group $(\mathrm{P}<0.05$; Fig. $2 \mathrm{C})$.

miR-302d inhibits apoptosis and promotes migration in HCC cells. The effect of miR-302d on apoptosis was assessed using fluorescence-activated cell sorting. The results demonstrated that cells overexpressing miR-302d exhibited a significant decrease in the percentage of cells undergoing apoptosis compared with $\mathrm{NC}(\mathrm{P}<0.001)$, whereas cells expressing miR-302d inhibitors exhibited a significant increase in the percentage of apoptotic cells $(\mathrm{P}<0.001$; Fig. 3A). In vitro migration assays indicated that miR-302d mimics significantly promoted the motility of SMMC-7721 cells $(\mathrm{P}<0.001)$, whereas inhibition of miR-302d significantly suppressed the motility of SMMC-7721 cells $(\mathrm{P}<0.001$; Fig. 3B).

miR-302d promotes xenograft tumor growth in vivo. To further determine the function of miR-302d, its effect on tumorigenicity was examined using a mouse model. Mice injected with SMMC-7721 cells stably expressing miR-302d inhibitors exhibited tumors that grew more slowly than those of mice injected with NC. These differences were significantly increased until the end of the evaluation period at 6 weeks $(\mathrm{P}<0.05$; Fig. 4A and B). Tumor weights were consistently decreased in miR-302d inhibitor expressing tumors compared with the controls $(\mathrm{P}<0.05$; Fig. 4C). These data indicate that miR-302d may promote tumor growth in vivo.

miR-302d directly targets TGFBR2 in HCC cells. To explore the regulation mechanism of miR-302d, TargetScan bioinformatics 
$\mathbf{A}$

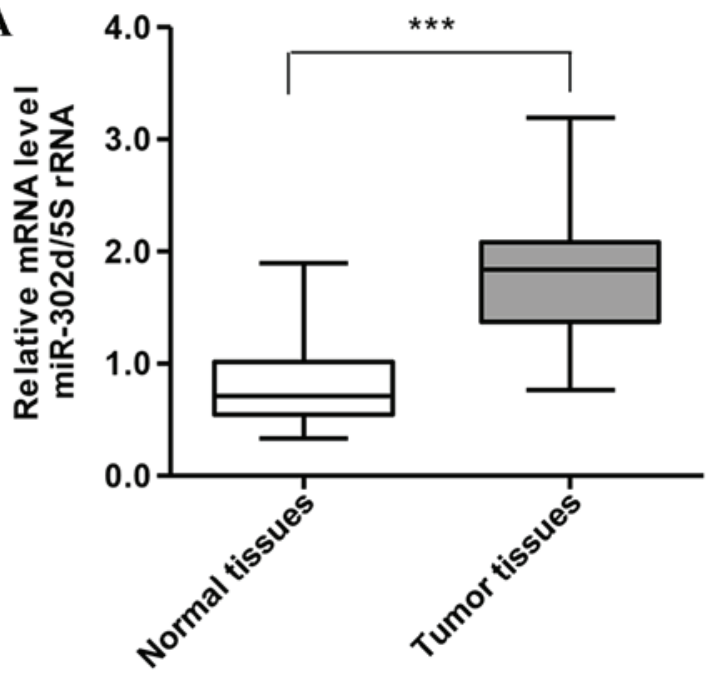

B

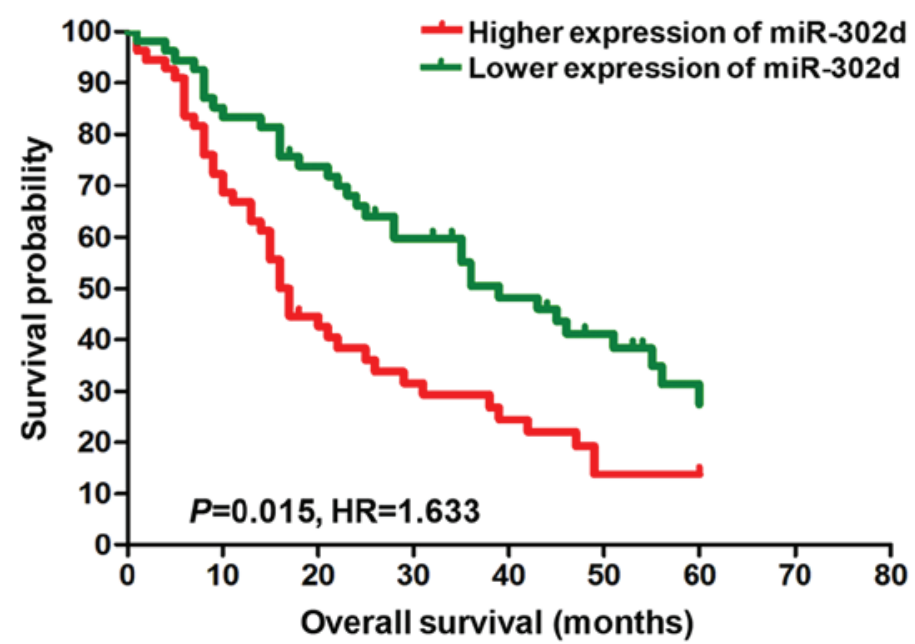

Figure 1. Association between miR-302d expression and survival time of patients with HCC. (A) Analysis of miR-302d expression levels in 30 pairs of HCC samples and adjacent healthy tissue by reverse transcription-quantitative polymerase chain reaction. miR-302d expression was normalized to 5S. (B) Effect of levels of miR-302d expression on the overall survival of patients with HCC. The cut-off level was set at the median value of the miR-302d expression levels in $110 \mathrm{HCC}$ patients. The group of patients with high miR-302d expression experienced poorer prognoses than that of the group with low miR-302d expression. ${ }_{* * * *} \mathrm{P}<0.001$. miR, microRNA; HCC, hepatocellular carcinoma; HR, hazard ratio.

A

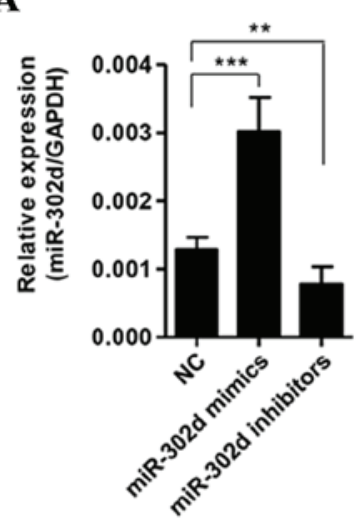

C

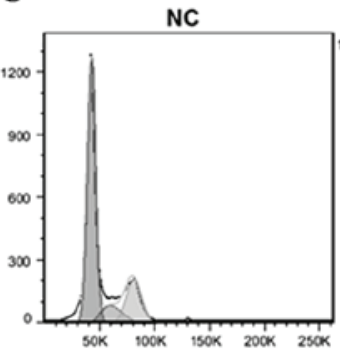

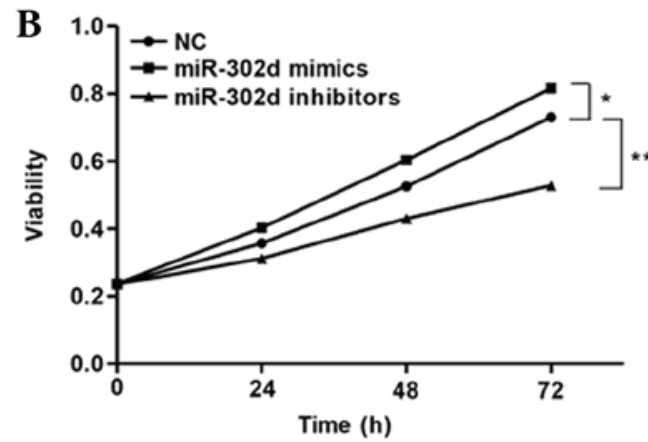

Figure 2. miR-302d promoted hepatocellular carcinoma cell growth and migration. (A) Relative expression levels of miR-302d were measured using reverse transcription-quantitative polymerase chain reaction in SMMC-7721 cells following transfection of miR-302d mimics, inhibitors or NC. (B) Cell viability was determined in SMMC-7721 cells transfected with miR-302d mimics, inhibitors or NC by CCK-8 assays. (C) The proportion of cells in each phase of the cell cycle was determined in SMMC-7721 cells following transfection of miR-302d mimics, inhibitors or NC by FACS. Histogram indicates the percentage of cells at the $\mathrm{G}_{0} / \mathrm{G}_{1}, \mathrm{~S}$ and $\mathrm{G}_{2} / \mathrm{M}$ phases. ${ }^{*} \mathrm{P}<0.05,{ }^{* *} \mathrm{P}<0.01,{ }^{* * *} \mathrm{P}<0.001$. NC, negative control; FACS, fluorescence-activated cell sorting; miR, microRNA; CCK-8, cell counting kit-8.

analysis (www.targetscan.org) was employed. TargetScan identified that TGFBR 2 mRNA contained potential binding sites of miR-302d (Fig. 5A). To confirm that TGFBR2 was a target of miR-302d and regulated by it in HCC cells, the TGFBR2 3'-UTR was cloned and inserted into a luciferase reporter vector. The luciferase assay indicated that miR-302d significantly suppressed luciferase activity in the vector containing the TGFBR2 3'-UTR ( $\mathrm{P}<0.01$; Fig. 5B). Western blot analysis demonstrated that miR-302d overexpression significantly suppressed endogenous TGFBR2 expression $(\mathrm{P}<0.001)$, 
A

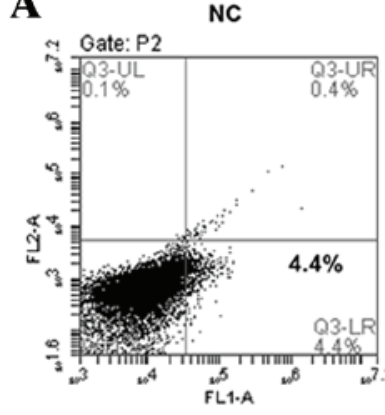

B

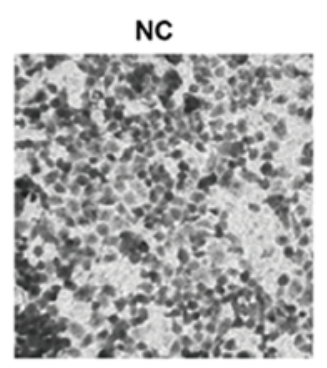

miR-302d mimics

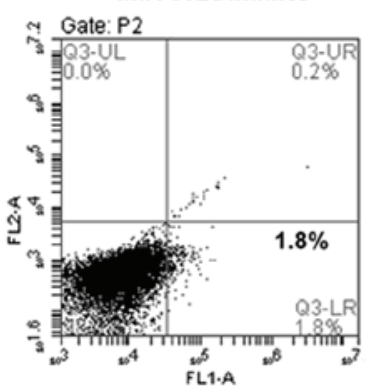

miR-302d mimics

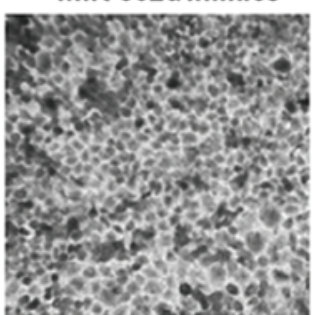

miR-302d inhibitors

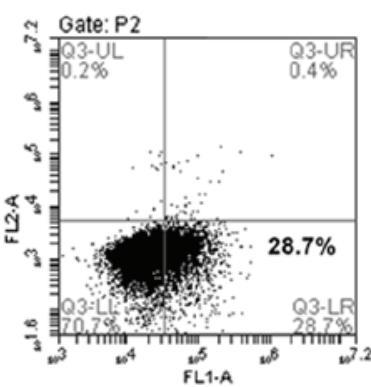

miR-302d inhibitors

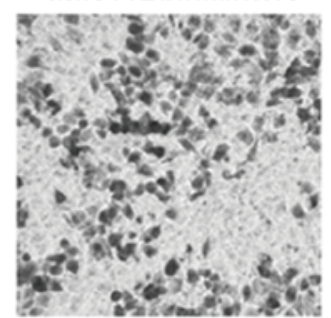

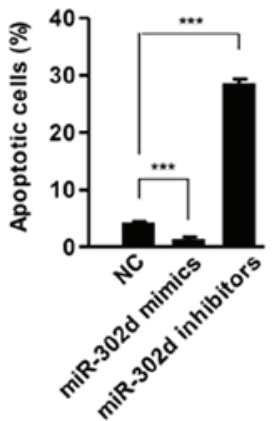

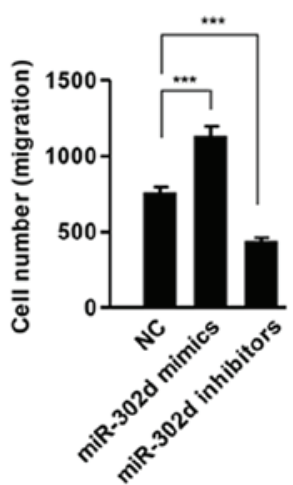

Figure 3. miR-302d inhibited apoptosis. (A) Apoptosis was determined in SMMC-7721 cells following transfection of miR-302d mimics, inhibitors or NC by FACS. Histogram indicates the percentage of cells at apoptosis. (B) Cell migration abilities were determined in SMMC-7721 cells transfected with miR-302d mimics, inhibitors or NC by Transwell assay. Magnification, $\mathrm{x} 200 .{ }^{* * * *} \mathrm{P}<0.001$. NC, negative control; FACS, fluorescence-activated cell sorting; miR, microRNA; CCK-8, cell counting kit-8.

A

B

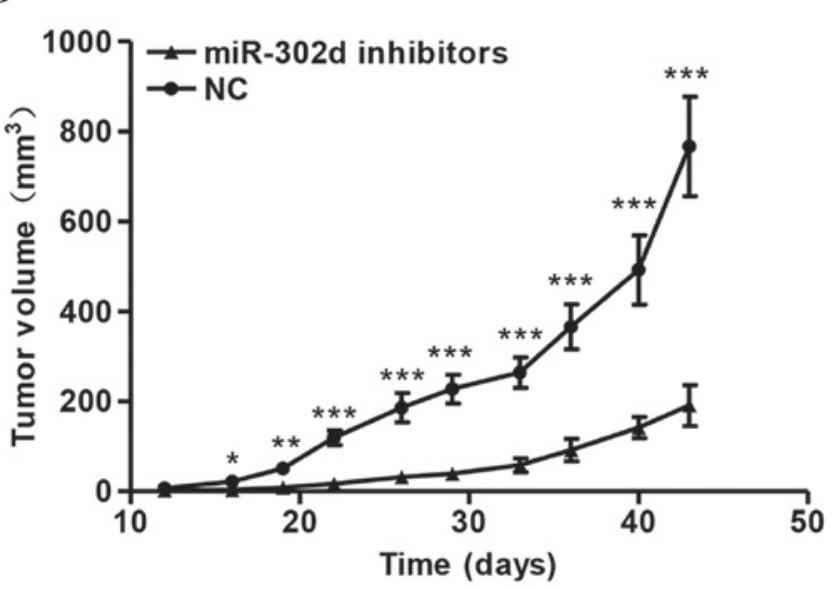

C

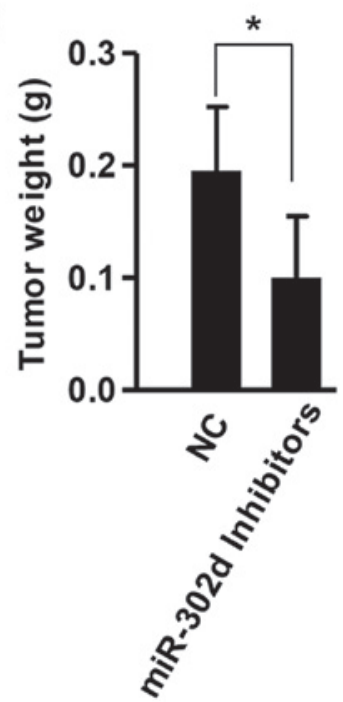

Figure 4. miR-302d inhibitors suppressed tumor growth in vivo. (A) Xenograft tumors from nude mice injected with SMMC-7721 cells stably expressing miR-302d inhibitors or NC. (B) Growth curve of tumor volumes. (C) Tumor weights. ${ }^{*} \mathrm{P}<0.05,{ }^{* * *} \mathrm{P}<0.01$ and ${ }^{* * * *} \mathrm{P}<0.001$. miR, microRNA; NC, negative control. 
A

\section{TGFBR2 3'-UTR Has-miR-302d}

B

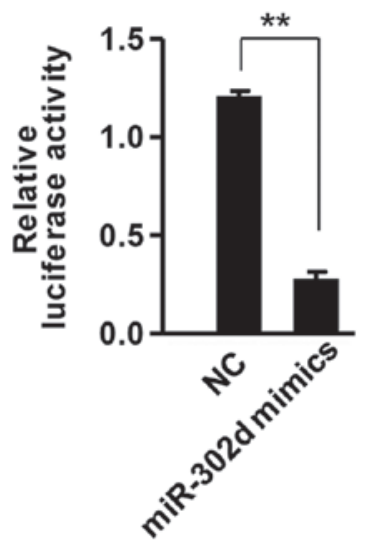

5'-TATATCAAAAGTCTCAAGCACTTA-3' 3'-UGTGAGUUUGUACCUUCGUGAAU-5'

C

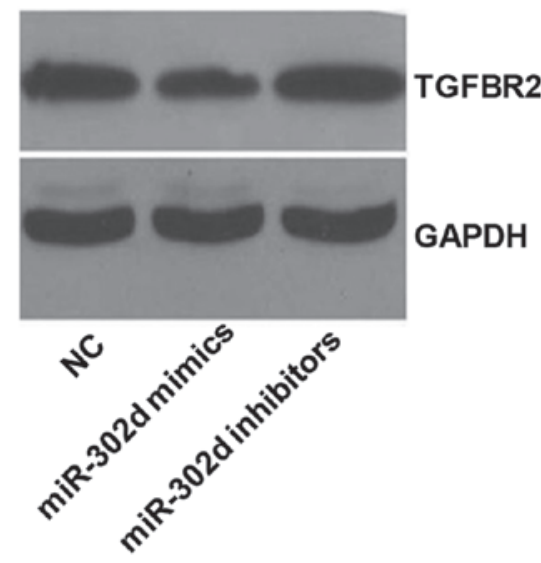

D

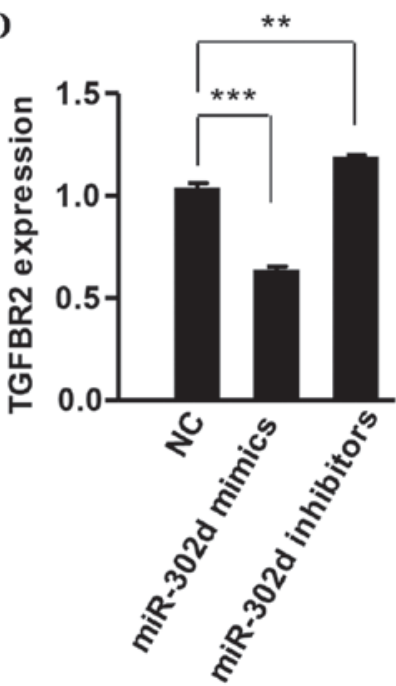

Figure 5. miR-302d directly targeted TGFBR2 in hepatocellular carcinoma cells. (A) Base-pairing interaction between miR-302d seed sequences and TGFBR2, as predicted by TargetScan. (B) Luciferase assay. SMMC-7721 cells were co-transfected with pGL3-TGFBR2 and miR-302d mimics, inhibitors or NC. Firefly luciferase activity was normalized to Renilla luciferase activity. (C and D) TGFBR2 protein levels were examined in SMMC-7721 cells transfected with miR-302d mimics, inhibitors or NC. ${ }^{* *} \mathrm{P}<0.01$ and ${ }^{* * * *} \mathrm{P}<0.001$. miR, microRNA; TGFBR2, transforming growth factor beta type II receptor; NC, negative control; 3'UTR, 3' untranslated region.

whereas inhibition of miR-302d significantly increased levels of TGFBR2 protein in SMMC-7721 cells ( $\mathrm{P}<0.01$; Fig. 5C and D). These results suggest that TGFBR2 is a target of miR-302d in HCC cells.

\section{Discussion}

HCC is the third leading cause of cancer-associated mortality worldwide (1). However, the molecular mechanisms responsible for the development and progression of HCC remain largely unknown. It has been reported that miRNAs are associated with HCC development and progression and, according to their function in tumorigenesis, they may act as oncogenes or tumor suppressors (5). Various miRNAs are oncogenic in HCC, including miR-21, miR-221, miR-222 and miR-151; however it has been suggested that a number of miRNAs, including miR-29, miR-122, miR-125b, miR-101 and miR-139, may be tumor suppressive in HCC (17). It has previously been demonstrated that in HCC, miRNAs are involved in the regulation of cell survival and proliferation, angiogenesis and metastasis $(9,17)$.

In the present study, results from RT-qPCR indicated that miR-302d is highly expressed in HCC, and a correlation was observed between increased miR-302d expression and decreased patient survival time. The data collected suggest that miR-302d promotes cell proliferation and migration, suppresses cell apoptosis, affects the cell cycle distribution of HCC cells in vitro, enhances tumorigenicity in vivo and may be used as a prognostic indicator of survival in patients with HCC. Therefore, miR-302d may be a novel oncogene in HCC.

miR-302d belongs to the miR-302 family, which consists of four highly homologous miRNA members, including miR-302c, miR-302b, miR-302d, mi-302a and miR-367 (18). It has been suggested that the expression of different forms of miR-302 may be associated with tumor progression $(19,20)$. Furthermore, it has been identified that miR-302b suppresses the proliferation of HCC SMMC-7721 cells (21). However, it has also been demonstrated that miR-302d increases cell proliferation and inhibits cell death in human adipose tissue-derived mesenchymal stem cells (13). Therefore, miR-302d may promote tumor growth. This is consistent with results from the present study that implicate miR-302d as a novel oncogene in HCC.

Furthermore, the mechanism by which miR-302d influences the development of HCC was investigated. TGFBR2 was predicted to be the potential target gene of miR-302d by bioinformatic algorithms. A luciferase activity assay indicated that miR-302d directly targeted the 3'-UTR of TGFBR2 mRNA. In addition, miR-302d overexpression significantly downregulated the expression of TGFBR2. These results suggest that miR-302d may function as a novel oncogene in $\mathrm{HCC}$ and contribute to tumor progression in $\mathrm{HCC}$.

TGF- $\beta$ signaling is tumor suppressive in numerous different cell types (22). Reduced expression or loss of the TGF $\beta$ type I and type II receptors (TGFBR1 and TGFBR2) has been reported in various types of cancer, including HCC (23-25). TGFBR2 has been identified as the direct target of numerous miRNAs, including miR-655, miR-520c, miR-373 and miR-211 (26). The results of the present study suggest that TGFBR2 is a novel target of miR-302d in HCC and may partially explain the mechanism by which miR-302d functions in HCC, and why TGFBR2 expression is reduced in HCC.

In conclusion, the results of the present study provide novel evidence that miR-302d negatively regulates TGFBR 2 expression, promotes HCC cell growth and migration, suppresses cell apoptosis and affects cell cycle distribution in vitro, and promotes tumorigenicity in vivo. Therefore, miR-302d may be a novel tumor promoter in HCC and may be developed as a novel therapeutic strategy to treat patients with HCC. 


\section{References}

1. He J, Gu D, Wu X, Reynolds K, Duan X, Yao C, Wang J, Chen CS, Chen J, Wildman RP, et al: Major causes of death among men and women in China. N Engl J Med 353: 1124-1134, 2005.

2. Parkin DM, Bray F, Ferlay J and Pisani P: Estimating the world cancer burden: Globocan 2000. Int J Cancer 94: 153-156, 2001.

3. Altekruse SF, McGlynn KA and Reichman ME: Hepatocellular carcinoma incidence, mortality, and survival trends in the United States from 1975 to 2005. J Clin Oncol 27: 1485-1491, 2009.

4. Nguyen VT, Law MG and Dore GJ: Hepatitis B-related hepatocellular carcinoma: Epidemiological characteristics and disease burden. J Viral Hepat 16: 453-463, 2009.

5. Esquela-Kerscher A and Slack FJ: Oncomirs-microRNAs with a role in cancer. Nat Rev Cancer 6: 259-269, 2006.

6. Varnholt H, Drebber U, Schulze F, Reynolds K, Duan X, Yao C, Wang J, Chen CS, Chen J and Wildman RP: MicroRNA gene expression profile of hepatitis $C$ virus-associated hepatocellular carcinoma. Hepatology 47: 1223-1232, 2008.

7. Ji J, Shi J, Budhu A, Yu Z, Forgues M, Roessler S, Ambs S, Chen Y, Meltzer PS, Croce CM, et al: MicroRNA expression, survival, and response to interferon in liver cancer. N Engl J Med 361: 1437-1447, 2009.

8. Wei R, Huang GL, Zhang MY, Li BK, Zhang HZ, Shi M, Chen XQ, Huang L, Zhou QM, Jia WH, et al: Clinical significance and prognostic value of microRNA expression signatures in hepatocellular carcinoma. Clin Cancer Res 19: 4780-4791, 2013.

9. Sun J, Lu H, Wang X and Jin H: MicroRNAs in hepatocellular carcinoma: Regulation, function, and clinical implications. ScientificWorldJournal 2013: 924206, 2013.

10. Li SS, Yu SL, Kao LP, Tsai ZY, Singh S, Chen BZ, Ho BC, Liu YH and Yang PC: Target identification of microRNAs expressed highly in human embryonic stem cells. J Cell Biochem 106: 1020-1030, 2009.

11. Dolezalova D, Mraz M, Barta T, Plevova K, Vinarsky V, Holubcova Z, Jaros J, Dvorak P, Pospisilova S and Hampl A: MicroRNAs regulate $\mathrm{p} 21$ (Waf1/Cip1) protein expression and the DNA damage response in human embryonic stem cells. Stem Cells 30: 1362-1372, 2012.

12. Borgdorff V, Lleonart ME, Bishop CL, Fessart D, Bergin AH, Overhoff MG and Beach DH: Multiple microRNAs rescue from Ras-induced senescence by inhibiting p21(Waf1/Cip1). Oncogene 29: 2262-2271, 2010.

13. Kim JY, Shin KK, Lee AL, Kim YS, Park HJ, Park YK, Bae YC and Jung JS: MicroRNA-302 induces proliferation and inhibits oxidant-induced cell death in human adipose tissue-derived mesenchymal stem cells. Cell Death Dis 5: e1385, 2014.
14. Wang Y, Zhao L, Xiao Q, Jiang L, He M, Bai X, Ma M, Jiao X and Wei M: miR-302a/b/c/d cooperatively inhibit BCRP expression to increase drug sensitivity in breast cancer cells. Gynecol Oncol 141: 592-601, 2016.

15. Livak KJ and Schmittgen TD: Analysis of relative gene expression data using real-time quantitative PCR and the 2(-Delta Delta C(T)) Method. Methods 25: 402-408, 2001.

16. Lewis BP, Burge CB and Bartel DP: Conserved seed pairing, often flanked by adenosines, indicates that thousands of human genes are microRNA targets. Cell 120: 15-20, 2005.

17. Wong CM, Kai AK, Tsang FH and Ng IO: Regulation of hepatocarcinogenesis by microRNAs. Front Biosci (Elite Ed) 5: 49-60, 2013.

18. Lin SL, Chang DC, Chang-Lin S, Lin CH, Wu DT, Chen DT and Ying SY: Mir-302 reprograms human skin cancer cells into a pluripotent ES-cell-like state. RNA 14: 2115-2124, 2008.

19. Barroso-delJesus A, Romero-López C, Lucena-Aguilar G, Melen GJ, Sanchez L, Ligero G, Berzal-Herranz A and Menendez P: Embryonic stem cell-specific miR302-367 cluster: Human gene structure and functional characterization of its core promoter. Mol Cell Biol 28: 6609-6619, 2008.

20. Card DA, Hebbar PB, Li L, Trotter KW, Komatsu Y, Mishina Y and Archer TK: Oct4/Sox2-regulated miR-302 targets cyclin D1 in human embryonic stem cells. Mol Cell Biol 28: 6426-6438, 2008

21. Wang L, Yao J, Shi X, Hu L, Li Z, Song T and Huang C: MicroRNA-302b suppresses cell proliferation by targeting EGFR in human hepatocellular carcinoma SMMC-7721 cells. BMC Cancer 13: 448, 2013.

22. Massagué J, Blain SW and Lo RS: TGFbeta signaling in growth control, cancer, and heritable disorders. Cell 103: 295-309, 2000

23. Ikushima $\mathrm{H}$ and Miyazono K: TGFbeta signalling: A complex web in cancer progression. Nat Rev Cancer 10: 415-424, 2010.

24. Bjerke GA, Pietrzak K, Melhuish TA, Frierson HF Jr, Paschal BM and Wotton D: Prostate cancer induced by loss of Apc is restrained by TGF $\beta$ signaling. PLoS One 9: e92800, 2014.

25. Mamiya T, Yamazaki K, Masugi Y, Mori T, Effendi K, Du W, Hibi T, Tanabe M, Ueda M, Takayama T and Sakamoto M: Reduced transforming growth factor-beta receptor II expression in hepatocellular carcinoma correlates with intrahepatic metastasis. Lab Invest 90: 1339-1345, 2010.

26. Harazono Y, Muramatsu T, Endo H, Uzawa N, Kawano T, Harada K, Inazawa J and Kozaki K: miR-655 is an EMT-suppressive microRNA targeting ZEB1 and TGFBR2. PLoS One 8: e62757, 2013. 